\title{
AN ONTOLOGY FOR SEMANTIC MODELLING OF VIRTUAL WORLD
}

\author{
Mezati Messaoud ${ }^{1}$, Foudil Cherif ${ }^{2}$, Cédric Sanza $^{3}$ and Véronique Gaildrat ${ }^{4}$ \\ ${ }^{1}$ Department of Computer Science, University of Ouargla, Algeria \\ ${ }^{2}$ Department of Computer Science, University of Biskra, Algeria \\ ${ }^{3}$ IRIT, Toulouse, France \\ ${ }^{4}$ IRIT, Toulouse, France
}

\begin{abstract}
This article presents a new representation of semantic virtual environments. We propose to use the ontology as a tool for implementation. Our model, called SVHsIEVs ${ }^{1}$ provides a consistent representation of the following aspects: the simulated environment, its structure, and the knowledge items using ontology, interactions and tasks that virtual humans can perform in the environment. In SVHsIEVs, we find two type of ontology: the global ontology and the local ontology for Virtual Human. Our architecture has been successfully tested in $3 D$ dynamic environments.
\end{abstract}

\section{KEYWORDS}

Virtual environments, semantic modeling, ontology, Virtual Human

\section{INTRODUCTION}

Current virtual environment representations describe models of environments so that browsers can effectively visualize their geometry and can support low-level interactivity in most of cases [1][2][3]. There is a gap between the low-level representation of the universe and how we conceptualize (and therefore how we think and talk about this universe). Thus, a high-level model's representation (including semantic descriptions of objects in the environment) is desirable in order to support user interactions which are richer in a more abstract level (querying for contents) and reasoning of deployed agents on the environments they inhabit.

We believe that the semantic information in virtual worlds should be considered as a component of the universe. Therefore, the construction of virtual environments should contain semantic annotations about the environment [1][4]. Modeling techniques are rooted in semantic information systems, namely databases, geographic information systems, etc. Data representation is a critical issue for these systems. Traditional approaches simply focus on techniques that support efficient storage and retrieval of data. Otherwise, semantic modeling makes the data meaningful, and therefore machine-readable [5].

The role of the semantic modeling of virtual environments (VEs) is to provide an abstract, highlevel and semantic description of different aspects of a VE: structure of the virtual environment, behaviors and interactions of entities, domain knowledge, etc.[2,3]. A main motivation for adding a semantic model is to ease the design of intelligent VEs. This intelligence of VEs mixed with

\footnotetext{
${ }^{1}$ Semantic Virtual Humans In Virtual Environments
}

DOI : 10.5121/ijaia.2015.6105 
International Journal of Artificial Intelligence \& Applications (IJAIA), Vol. 6, No. 1, January 2015

artificial agents and users can be defined as the capacity of artificial agents to exhibit human-like behaviors and to be capable to assist users to solve a specific problem [5].

Ontologies have been used in virtual worlds as a relevant formalism to provide a conceptual representation of scene contents. The main idea has been a direct mapping between graphical contents and ontologies [2,6]. The concepts in an ontology can be the exact copy of the specific graphical resources, but this leads to several inconveniences. First, ontologies are not able to represent entities with no graphical representation in VEs. Second, it is not possible to share common properties among a family of graphical resources. For instance, movable objects have some properties in common in comparison with unmovable objects.

In short, our model proposes that each virtual object in a particular virtual environment is geometrically considered as its minimum bounding-box, and corresponds to a particular type of object in a specific ontology. In addition, this model also establishes relationships between elements in the VE. This model (called SVHsIEVs) uses virtual humans in VE. Each virtual human uses both two techniques. The first technique: Guidelines, ensures coherence and sequence of tasks. The second technique: Querying ensures the communications between agents and ontology.

In the next section, we briefly review related works. A detail description of the proposed semantic modeling is given in section 3. Section 4 describes the definition and the role the ontology in VEs. The architecture of the proposed environment is illustrated in section 5. Section 6 concludes the paper and outlines some future works.

\section{RELATED WORKS}

This section describes several approaches and concrete works concerning the addition of a semantic level to a virtual world, and designing of VE along with the semantic model. Semantic modelling is expected to offer a richer and more expressive representation of VEs. There are three main approaches for the semantic modelling of VEs. First approach: Designing the VE along with the semantic model. The metadata are added in the model as the objects are created. This technique has been used either for content-oriented and system-oriented approaches. Second approach: Building the VE based on a pre-existent semantic level. The main idea of this technique is to get benefits from an existing semantic model. For instance, one can use an existing Geographical Information System to build virtual urban environments. Third approach: Adding semantic annotations to the pre-existent VE. The semantic annotations can be multimedia resources, such as texts, images, sounds, and Web links. In this case, the added information makes sense only for the user, but is not semantically interpreted by the system.

Designing the VE along with the semantic model. The metadata are added in the model as the objects are created. This technique has been used either for content-oriented and system-oriented approaches. Cavazza \& Ian [8] Recognized a need for acknowledge presentation layer for virtual environments. Thalmann et al [9] Introduced the concept of annotated environment (with structured representations of its contents and purpose) Agent architecture for interacting with the annotated environment. Chang at all [13], presented a Framework With an ontology-based cognitive middle layer minds and the environment manage semantic concepts This layer also represents action through Casual Rules. With Grimaldo et al [14], an ontology is used to define social relations among agents with in an artificial society.

Building the VE based on a pre-existent semantic level. The main idea of this technique is to get benefits from an existing semantic model. For instance, one can use an existing Geographical 
International Journal of Artificial Intelligence \& Applications (IJAIA), Vol. 6, No. 1, January 2015

Information System to build virtual urban environments. According to Ishida [27] the design of digital cities follows a general three layer model. Its layers are as follows: information layer, where digital archives, web pages and real-time sensory data from physical cities are integrated and reorganised using the city metaphor; interface layer, where graphical representations provide a view of the city; interaction layer, where resident and tourist interact with each other.

Adding semantic annotations to the pre-existent VE. The semantic annotations can be multimedia resources, such as texts, images, sounds, and Web links. In this case, the added information makes sense only for the user, but is not semantically interpreted by the system. In this sense, voice annotation systems are proposed in the Virtual Book project [26].

Doyle [4] introduces the concept of the annotated environment, so the structured representation of their content and their objectives are available to any agent in the environment. This description of an agent architecture gives the possibility to interact with an annotated virtual environment, with a structure for representing information in these environments.

Badawi\& Donikian [10] describe the STARFISH (synoptic objects for tracking actions received from interactive surfaces and humanoids) architecture that uses synoptic objects to allow realtime object manipulation by autonomous agents in a virtual environment. A set of actions is defined. Then these actions are assigned to interactive surfaces that define the geometry of an object and that are concerned by the action. The agent then uses these interactive surfaces to get the data specific to the object when it wants to manipulate it and to adapt its behavior accordingly.

The current trend is the use of ontologies to model the semantic information of virtual environments. Vanacken et al [11] introduce the use of semantic information, represented using ontologies, in conceptual modelling of interaction invirtual environments.

This semantic information itself is created during the design of the virtual world. More concretely, semantics is incorporated in NiMMiT (Notation for MultiModal interaction Techniques) [12], a diagram based notation intended to describe multimodal interaction. Some works have proposed complete architectures that include a semantic layer, which is the interface between the agents and the world. This layer usually models the world through a semantic representation defined according to a set of ontologies.

The ontology is considered as a means for social relations between agents within an artificial society. These relations must be used into account in order to display socially acceptable decisions [15].

This approach has been used to simulate the virtual bar of a university, where groups of waiters and customers interact with both the objects in the scene and the other virtual agents finally displaying complex social behaviors [16].

Ibânêz et al [17] think that application approaches are necessary, and they are different from one another in nature. Thus, the model they proposed is situated at a lower level than approaches, which depend of applications. Their model does not intend to substitute to application dependent approaches, but to constitute a common lower level for all of them. The authors'intention was to create a useful model, that is, a model actually employed by the world creators. Thus, their principle was that it should not require a great annotation effort from the environment creators. As a result, the model consists of a reduced number of different features, and the majority of them can be automatically annotated. 
Tutenel et al [18] introduced the Semantic Class Library to design semantic VEs, notably 3D games. After creating a 3D model, the designer associates the elements of the 3D model to existing classes in the library. Otherwise, the designer can create a new class with the desired properties. Beyond the 3D representations of objects within the game world, the Semantic Class Library provides additional semantics to the objects, such as physical attributes (e.g., the mass or material), functional information (e.g., how one can interact with an object).

\section{ONTOLOGY IN VIRTUAL ENVIRONMENT}

Virtual Humans are virtual entities with a rich set of functionalities and potential, present in a VE. One of the main instruments used to lay-down the foundations of a knowledge-based system are ontologies. Ontology defines a common vocabulary for domain-users (researchers or experts in a particular area) who need to share information in a particular domain. It includes machineinterpretable definitions of basic concepts in the domain and relations among them. The semantics-based representation can be enhanced by means of knowledge-management techniques and tools. One of the main tools used to lay-down the foundations of a knowledge-based system is therefore an ontology. A first one focuses on the adaptive multimodal interfaces system and a second one formalizes the knowledge related to the creation of virtual humans and serves as a basis for a general ontology for Virtual Environments [20]. Many consider W3C'sWeb Ontology Language (OWL) the prospective standard for creating ontologies on the Semantic Web.

OWL has three species: OWL Lite, OWL DL and OWL Full, in ascending order according to expressiveness. We can divide the use of ontologies in the domain of Virtual Environments into three uses; the first use: Ontologies in the context of Virtual Environments [21,22], the second use: Ontology for interactive Virtual Environments[23,24], the third use: Ontology for Virtual Humans $[19,25]$.

The conceptualization step is to identify a body in the knowledge domain and to clarify the conceptual nature (concepts, relations, properties and relations of concepts, rules, constraints, etc.)

The ontologization step consists in modeling in a formal language the domain properties, the objective is to obtain a model in which almost all the ambiguities inherent in natural language are lifted.

The operationalization is to make operational or functional ontology. First, must select the ontology language and the tool to build the ontology.

\section{OUR FRAMEWORK}

In the framework SVHsIEVs, we attempt to apply the influence of the integration semantic layer in virtual worlds. This semantic layer is distributed according to two levels. The first level is global; in this level we can define semantic information on a more global way. The second level concerns the virtual objects; in this level, objects need to transcend the geometry concepts and more abstract information need to be incorporated into the object's description. Many properties of real-world objects should be represented in their virtual counterparts to allow an algorithm to perform some kind of reasoning on objects (e.g., the physical attributes define whether or not the object is too heavy to carry, or the functional information is necessary to decide if an AI character can use the object to reach a goal). 
In our proposal semantic information use the ontology to describe the concepts used in the domain along with their properties and relations between them, in each two levels.

Our contribution is adding semantic information (contextual attributes and relationships between concepts) to virtual objects and with the aim to use this information to define and redefine interaction with environment.

As discussed earlier in this paper, the proposed framework is based on the integration of semantics information in virtual environment, and we show this integration in different layer. Also, for each objet in the environment composed of two aspects: geometry and semantic, the aspect semantic is based on contextual information only but using the relationship between different concepts, these concepts are present information's semantics of different objects in virtual environment.

In this paper we present a Semantic Virtual Environment approach that uses ontologies as an appropriate basis to animate virtual environments inhabited by intelligent virtual Humans. Figure 1 show the architecture of our multi-agent framework, which can be divided into several parts:

Ontologies define the world knowledge base as well as the set of all possible relations among the agents and virtual humans. We distinguish two levels of representation: the SVE Core Ontology is a unique base ontology suitable for all virtual environments which can be extended by different Domain Specific Ontologies in order to model application-specific knowledge. Then, environments can be constructed by instantiating the classes of these ontologies. For example, in section Implementation we will create pedestrians a virtual city with a large number of objects cars, crossroads, etc.

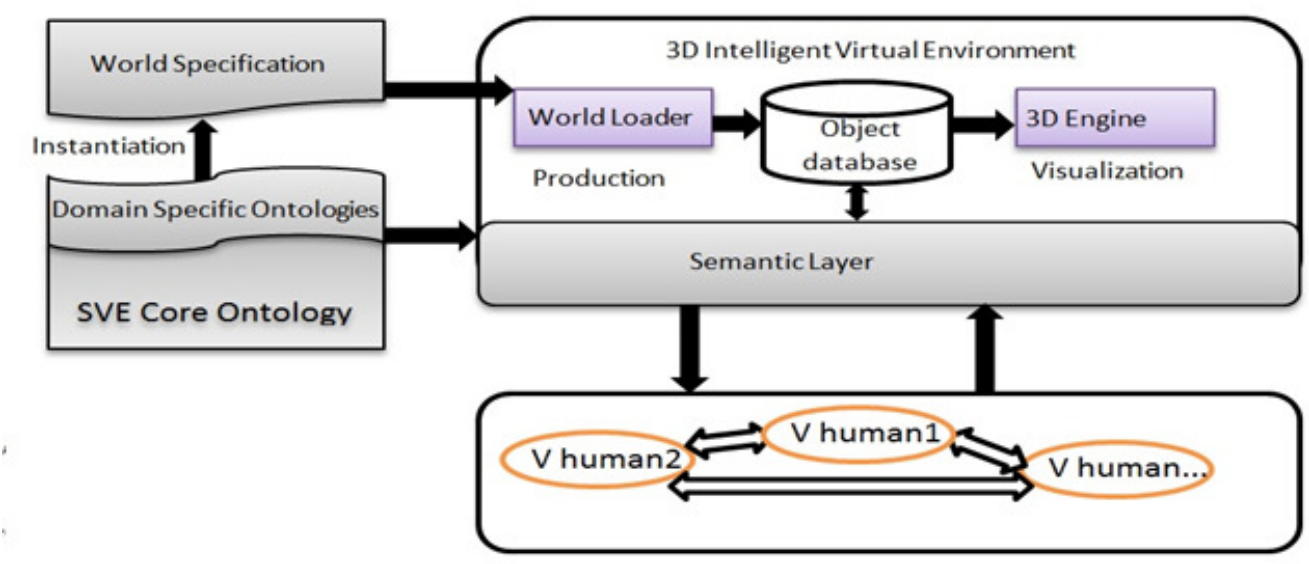

Figure 1. SVHsIEVs architecture

The Semantic Layer is the interface between the virtual human and the world. It uses the ontology so as to reduce the information flow; this layer is in charge of executing the actions requested by the agents as well as maintaining their semantic effects.

In the structure of Virtual Human we find the combination between three aspects: Virtual human intelligent (VHI), Querying and Guidelines. The VHI insures the intelligent interaction with world and the others VHI and the querying insures communication (asks and answers) with the world. The Guidelines insures the planning of different tasks. 


\subsection{Ontology of virtual environment}

The goal of ontology design for virtual environment in this architecture is two parts. First, we would like to keep the information that exists in the virtual environment such as object geometry and transformation. Second, we use semantic information about the virtual objects can facilitate the computation of advanced reasoning procedures such as a navigation in the world.

Our ontology design of the virtual environment is shown in Figure 2. The root of the ontology is the environment node, which contains world information (environment) and all the virtual objects (object) in the environment. In order to retain the semantic information of the virtual objects, we have designed the base Info and Transform nodes; in this node we show sub-information as position and rotation. Each object also has some additional attributes such as name, weight, height and tag. All this attributes are designed for public properties for virtual objects. For example, the urban environment, one can tag certain objects as s pedestrian area and car area such that these regions can be treated appropriately by the urban environment according to their meanings in the world. Each object in environment as (building, car and Column lights...) may also have the attribute of Approximation 3D, which is a polygon that can be used to define 3D approximation of obstacles in the environment for the navigation.

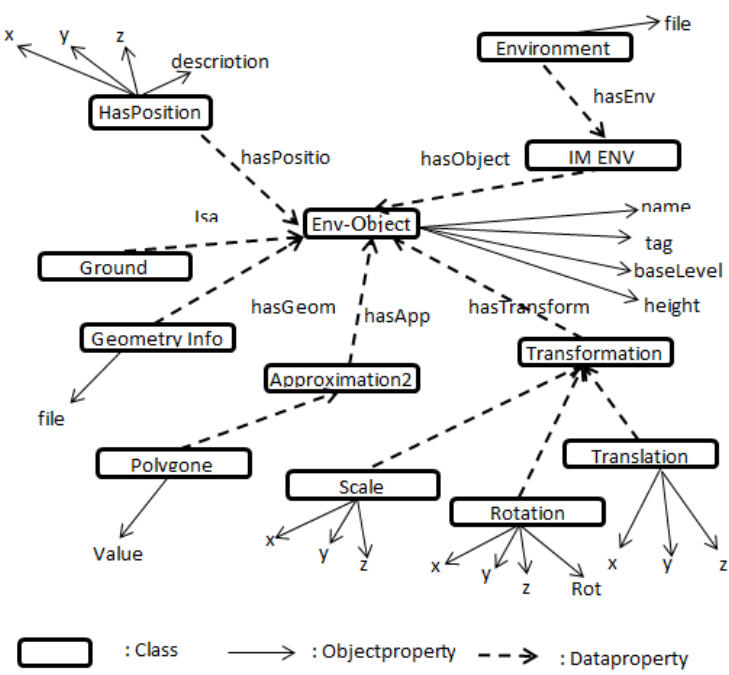

Figure 2. Ontology design for virtual environment

\subsection{VHs architecture}

The Virtual Human is based on three aspects; Virtual human intelligent (VHI), Querying and Guidelines. The VHI gives the intelligent interaction with world and other VHI and the querying gives asks and reception the answers with world. The Guidelines gives the pacification of different tasks.

The module gives the aspect querying, it is a communication module, and in this module we show two types of communication: the first protocols with other Virtual Human by message for example demand the service or information, the second protocol with environment by the queries. 
International Journal of Artificial Intelligence \& Applications (IJAIA), Vol. 6, No. 1, January 2015

The answer of these queries is divided into two cases according to natural answer; if the answer is static information like position or direction by example, in this case, the answer is simple processing; we take the query and search in the ontology of data information without treatment. In second case, we use different modules as reasoning module and ontology.

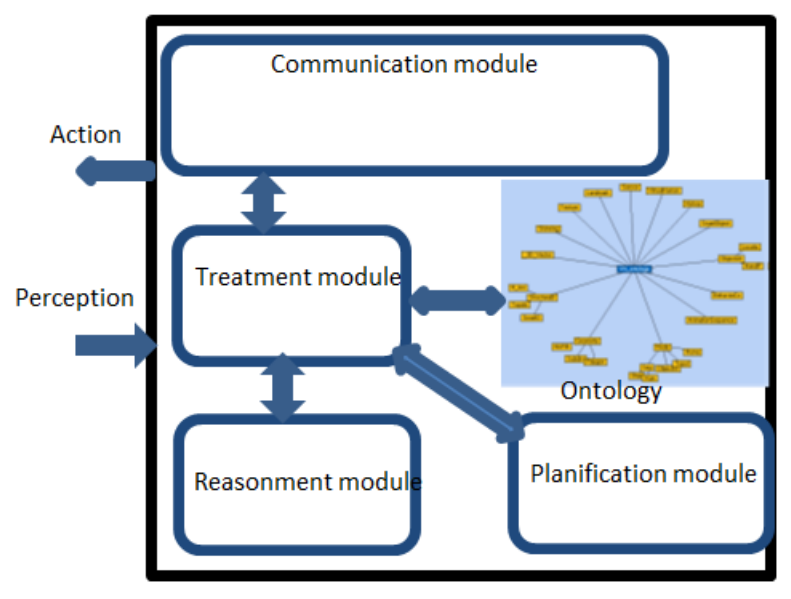

Figure 3. A Virtual Human architecture

\subsection{Ontology design for Virtual Human}

In our architecture, a VH has an ontology. In this ontology, we describe the basic ontology classes and attributes (as shown in Figure 4) that we have designed for the applications of the VH navigations. Although a VH is also an object in a virtual environment, they have more active and complicated roles to play. For example, a user in Anthropometry Description set attributes as Height, Wight, Age, Gender, Speed and Acuity. A VH may contain some basic attributes such as name, transform, and status. We also take the tired and stress for present the influence of these properties in speed and acuity of $\mathrm{VH}$.

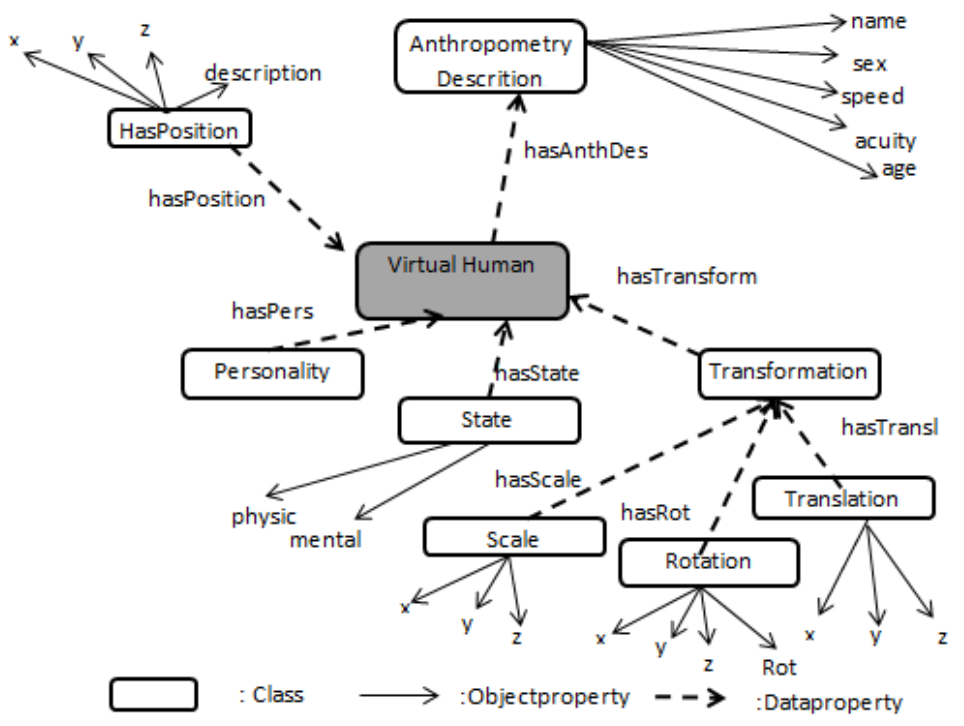

Figure 4. Ontology design for $\mathrm{VH}$ 


\section{Conclusion and Future Works}

The semantic information is an essential element for enabling richer contents and behaviors in the future development of virtual world. The concept of Ontology in SVHsIEVs has more functions such as stored and data management (add, up-date,...), our first parts build global ontology after adding and data classing according to the three phases (conceptualization, ontologization , operationalization )

Furthermore, the VH extracted from the ontology allows the VHs to reuse their information in different contexts. We will add different animation components owned by different VHs to interact with each other, will integrate more semantic information for $\mathrm{VH}$ ontology such as information of personality and physic and mental state, will implement our model with different dynamic environments.

\section{REFERENCES}

[1] Jesús Ibáñez\&Carlos Delgado-Mata, (2011)“Lessons from research on interaction with virtual environments". J. Network and Computer Applications 34(1),pp268-281.

[2] Martinez, A.G.R, (2009) "Semantics for Virtual Humans", Thèse No 4301, École Polytechnique Fédérale de Lausanne.

[3] Mario a. Gutierrez, (2005)“Semantic Virtual Environments”, Thèse, École Polytechnique Fédérale de Lausanne.

[4] Doyle P, (2002) "Believability through context using "knowledge in the world" to create intelligent characters", Proceedings of the first international joint conference on autonomous agents and multi agent systems. New York, NY, USA: ACM.

[5] Trinh T-H, (2012) "A Constraint-based Approach to Modelling Spatial Semantics of Virtual Environments", Université Européenne de Bretagne, PhD thesis, Brest, France.

[6] KIM, In-Cheol. KGBot, (2003) "A BDI agent deploying within a complex 3D virtual environment, Intelligent Virtual Agents. Springer Berlin Heidelberg, pp 192-196.

[7] Gao Z Q, (2003), "Designing Interactive Multi-agent Systems with Semantic 3D Environments", Proceedings of International Workshop on Semantic Web Technologies and Application Technologies, Nara, Japan.

[8] Cavazza M, Ian P, (1998). "High-level interpretation in dynamic virtual environments", Working notes: intelligent virtual environments, workshop at the 13th biennial European conference on artificial intelligence (ECAI-98). Brighton, UK.

[9] Thalmann D, Farenc N, Boulic R, (1999) "Virtual human life simulation and database: why and how", International symposium on database applications in non- traditional environments, DANTE'99.

[10 ]Badawi M, Donikian S,(2004) "Autonomous agents interacting with their virtual environment through synoptic objects", CASA 2004.

[11 ]Vanacken L, Raymaekers C, Coninx K, (2007) "Introducing semantic information during conceptual modelling of interaction for virtual environments", WMISI '07: proceedings of the 2007 workshop on multimodal interfaces in semantic interaction. New York, NY, USA: ACM; pp 17-24.

[12] Boeck JD, Raymaekers C, Coninx K,(2007)“Comparing NiMMiT and data-driven notations for describing multimodal interaction”, TAMODIA'06: proceedings of the fifth international conference on task models and diagrams for users interface design. Berlin, Heidelberg: Springer-Verlag;pp 21729.

[13] Chang PH-M, Chien Y-H, Kao EC-C, Soo V-W, (2005) "A knowledge based scenario framework to support intelligent planning characters".

[14] Grimaldo F, Barber F, Lozano M,(2006) "An ontology-based approach for IVE+VA", International conference on intelligent virtual environments and virtual agents (IVEVA). Aguascalientes, Mexico; pp 67-76.

[15] Grimaldo F, Lozano M, Barber F, (2008) “A multi agent framework to animate socially intelligent agents", Innovations in hybrid intelligent systems, pages17-24. 
International Journal of Artificial Intelligence \& Applications (IJAIA), Vol. 6, No. 1, January 2015

[16] Grimaldo F, Lozano M, Barber F, Vigueras G, (2008) "Simulating socially intelligent agents in semantic virtual environments", The Knowledge Engineering Review; 23(4): pp369-88.

[17] Ibânêz J, Delgado-MataC. (2006)"Abasics semantic common level for virtual environments", International Journal of Virtual Reality5(3), pp 25-32.

[18] Tutenel, T., Smelik, R., Bidarra, R., and Jan De Kraker, K, (2009)"Using semantics to improve the design of game worlds", Proceedings of the Fifth Artificial Intelligence and Interactive Digital Entertainment Conference, pp14-16.

[19] Gutiérrez M, Thalmann D, Vexo F, Moccozet L, Magnenat N, Spagnuolo M, (2005) "An Ontology of Virtual Humans: incorporating semantics into human shapes”, Proc. of Workshop towards Semantic Virtual Environments, pp57-67.

[20] Ian Horrocks, Peter F. Patel-Schneider, Deborah L. McGuinness, and Christopher A. Welty, (2007) "OWL: a Description Logic Based Ontology Language for the Semantic Web", In Franz Baader, Diego Calvanese, Deborah McGuinness, Daniele Nardi, and Peter F. Patel-Schneider, editors, The Description Logic Handbook: Theory, Implementation, and Applications (2nd Edition), chapter 14. Cambridge University Press.

[21] RuffaldiE, Evangelista C, and Bergamasco M. (2004) "Populating virtual environments using semantic web", Proceedings of 1st Italian Semantic Web Workshop: Semantic Web Applications and Perspectives.

[22] SotoM, Allongue S, (2002) "Modeling methods for reusable and interoperable virtual entities in multimedia virtual worlds", Multimedia Tools Appl., 16(1-2):161-177.

[23] Gruber T, (1991) "The role of a common ontology in achieving sharable, reusableknowledge bases", Proceedings of the Second International Conference onPrinciples of Knowledge Representation and Reasoning, pp 601-602.

[24] Gutiérrez M, Thalmann D, and Vexo F., (2005) "Semantic Virtual Environments with Adaptive Multimodal Interfaces" Multimedia Modelling Conference, pp 277-283.

[25] Garcia-Rojas A, Vexo F, Thalmann D, Raouzaiou A, Karpouzis K, Kollias S, (2006) "Emotional body expression parameters in virtual human ontology", 1st International Workshop on Shapes and Semantics, Matsushima, Japan,pp 63-70.

[26] Verlinden, J., Bolter, J., and Van der Mass, C. (1993). "Voice annotation: Adding verbal information to virtual environments". In Proceedings of the European Simulation Symposium, pages 60-69.

[27] Toru Ishida. (2000 )."Understanding Digital Cities, Digital Cities: Experiences, Technologies and Future Perspectives”, Lecture Note in Computer Science, vol 1765, Springer-Verlag.

\section{Short Biography}

\section{Mezati Messaoud}

Mezati Messaoud is a Phd student at University of Ouargla since 2009. He works in the LESIA Laboratory Biskra University. His current research interest is in artificial intelligence, artificial life, crowd simulation, behavioral animation and virtual characters.

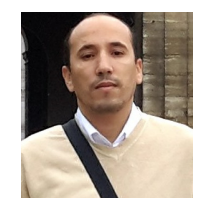

\section{Foudil Cherif}

Foudil Cherif is an associate professor in the department of Computer Sciences at Biskra University and head of LESIA Laboratory. He received his Phd in computer science from Biskra University in 2006. His first underground degree is Engineer in computer science from University of Constantine, Algeria in 1985. He received his Msc in computer science from Bristol University, UK in 1989. His researches focus primary on Behavioural animation, Crowd simulation, Autonomous agents, Artificial intelligence and software engineering.

\section{Cédric Sanza}

Cédric Sanza earned his $\mathrm{Ph}$. D. in 2001 and he is a research lecturer at University of Toulouse since 2002. He works in the field of simulation of virtual characters. He mainly focuses on physical motion and behavioral systems to design autonomous entities in 3D
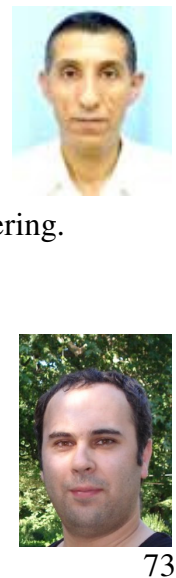
International Journal of Artificial Intelligence \& Applications (IJAIA), Vol. 6, No. 1, January 2015

worlds. He is also interested in learning by imitation in classifier systems to automatically build complex behaviors.

\section{Véronique Gaildrat}

Véronique Gaildrat is a full professor in the department of Computer Sciences at University of Toulouse since 2007. She is member of the IRIT laboratory (Institut de Recherche en Informatique de Toulouse) where she works in the field of declarative modeling of virtual environments. Lately she worked in the field of theater, in order to automatically create a virtual scenography based on the author's text, including representation of the emotional state of virtual actors.

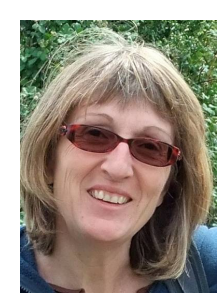

